\title{
UNITS AND PERIODIC JACOBI-PERRON ALGORITHMS IN REAL ALGEBRAIC NUMBER FIELDS OF DEGREE 3
}

BY

\author{
LEON BERNSTEIN
}

\begin{abstract}
It is not known whether or not the Jacobi-Perron Algorithm of a vector in $R_{n-1}, n \geqslant 3$, whose components are algebraic irrationals, always becomes periodic. The author enumerates, from his previous papers, a few infinite classes of real algebraic number fields of any degree for which this is the case. Periodic Jacobi-Perron Algorithms are important, because they can be applied, inter alia, to calculate units in the corresponding algebraic number fields. The main result of this paper is expressed in the following theorem: There are infinitely many real cubic fields $Q(w), w^{3}$ cubefree, $a$ and $T$ natural numbers, such that the Jacobi-Perron Algorithm of the vector $\left(w, w^{2}\right)$ becomes periodic; the length of the primitive preperiod is four, the length of the primitive period is three; a fundamental unit of $Q(w)$ is given by $e=a^{3} T+1-a w$.
\end{abstract}

0. Introduction. The problem of calculating a system of fundamental units in algebraic number fields of degree $n>2$ is still an open and challenging question. Dirichlet's famous theorem states only that the basis of the group of units in such fields is finite, and he also gives the exact number of elements of the basis of this group for a given field. But neither he nor his successors who developed the theory of algebraic numbers succeeded in stating a constructive method to calculate a system of fundamental units for an algebraic number field.

Jacobi [5] invented an algorithm for real cubic algebraic number fields which, as will be shown later, would have solved this problem. But he either did not know it, or was not concerned about units; so did Perron [8] who generalized Jacobi's algorithm for any real algebraic number field of degree $n \geqslant 3$. In honor of these two great mathematicians, the author [2(a)] has named this algorithm "the Jacobi-Perron Algorithm", abbreviated J. P. A., and has generalized it in [2(b)]. A breakthrough in finding a fundamental unit in (not totally) real numerical cubic algebraic number fields started with Voronoi [10] whose algorithm was based on ideas of Minkowski. His algorithm was recently generalized by Bilevich [4]. London and Finkelstein [6] applied Voronoi's and Bilevich's algorithm to the solution of Mordell's equation $y^{2}-k=x^{3}$. An important algorithm for the calculation 
of units in algebraic number fields is due to Bergman [1]. Significant contributions to the theory of units in rings of algebraic number fields have been made by Zassenhaus [11].

In this paper the author states a class of infinitely many (not totally) real cubic algebraic number fields whose bases become periodic by the J. P. A. and states, in a simple explicit form, a fundamental unit which is calculated from the period or preperiod of the J.P.A. Since some readers may not be familiar with the J.P. A., it shall be recalled in the following

Definition. Let $a^{(0)}$ be a vector in the real Euclidean vector space $R_{n-1}$, viz.

$$
a^{(0)}=\left(a_{1}^{(0)}, a_{2}^{(0)}, \ldots, a_{n-1}^{(0)}\right) \quad\left(n \geqslant 2, a_{i} \in R_{1}, i=1, \ldots, n-1\right) .
$$

A sequence $\left\langle a^{(v)}\right\rangle$ of vectors in $R_{n-1}$ is called the J. P. A. of $a^{(0)}$, if

$$
\begin{gathered}
a^{(v+1)}=\left(a_{1}^{(v)}-b_{1}^{(v)}\right)^{-1}\left(a_{2}^{(v)}-b_{2}^{(v)}, \ldots, a_{n-1}^{(v)}-b_{n-1}^{(v)}, 1\right) \\
\quad(v=0,1, \ldots), \\
a_{1}^{(v)} \neq b_{1}^{(v)} ; b_{i}^{(v)}=\left[a_{i}^{(v)}\right] \quad(i=1, \ldots, n-1) ;
\end{gathered}
$$

$[x]$ is the greatest rational integer contained in $x$.

Jacobi and Perron called their algorithms "periodic", if there exist nonnegative rational integers $l, m$ with $l \geqslant 0, m \geqslant 1$, such that

$$
a^{(v)}=a^{(v+m)} \quad(v=l, l+1, \ldots) .
$$

For $\min l$ and $\min m$ the sequences $a^{(0)}, a^{(1)}, \ldots, a^{(l-1)}$ and $a^{(l)}, a^{(l+1)}, \ldots$, $a^{(l+m-1)}$ are called respectively the "primitive preperiod" and the "primitive period" of the J. P. A., $l$ and $m$-their lengths. If $l=0$ the J.P. A. is called "purely periodic". The main result of this paper is stated in the following

THEOREM. Let

$$
m=a^{6} T^{3}+3 T\left(a^{3} T+1\right) ; \quad a, T \in N ; m \text { cubefree. }
$$

Let $Q(w), w^{3}=m$, be the real cubic field generated by adjunction of $w$ to the field $Q$ of rationals. Then the J. P. A. of $a^{(0)}=\left(w, w^{2}\right)$ is period; the length of the primitive preperiod of this $J . P$. A. equals $l=4$, the length of its primitive period equals $m=3$. A fundamental unit of $Q(w)$ is given by

$$
e=1+a^{3} T-a w
$$

The reader should note that $w, w^{2}$, as they appear in $a^{(0)}$, is not a basis of $Q(w)$; such would be given by $1, w, w^{2}$. Jacobi and Perron used the homogeneous notation $a^{(0)}=\left(1, w, w^{2}\right)$, while the author uses the nonhomogeneous notation $a^{(0)}=\left(w, w^{2}\right)$. 
The author is deeply indebted to the referee for his most valuable improvements and corrections of this paper.

1. Previous results of the author. In [8] Perron proved that if the J. P. A. of a vector $a^{(0)}=\left(a_{1}^{(0)}, \ldots, a_{n-1}^{(0)}\right)$ becomes periodic, then the components $a_{1}^{(0)}, \ldots, a_{n-1}^{(0)}$ belong to an algebraic number field of degree $\leqslant n$, and if they are linearly independent, the degree of that field is exactly $n$. The inverse problem, whether the J. P. A. of a vector $a^{(0)}$ whose components belong to an algebraic number field of degree $n$ always becomes periodic, is still challengingly open. Only a few numerical examples of periodic J. P. A. for real cubic algebraic number fields were known. It was not until some fifteen years ago, that the author succeeded in finding a few infinite classes of algebraic number fields, so that the J. P. A. of a vector $a^{(0)}$ with components properly chosen from these fields becomes periodic. We shall enumerate shortly these periodic J. P. A.'s.

(1) In [2(c)] the author proved: Let $w^{n}=D^{n}+d ; n \geqslant 2, D, d \in N ; D \geqslant$ $(n-2) d$. Then the J. P. A. of the vector $a^{(0)}=\left(w, w^{2}, \ldots, w^{n-1}\right)$ becomes periodic; the length of the primitive preperiod $l=n-1$, the length of the primitive period $m=n$ if $d \neq 1$, and $m=1$ if $d=1$.

(2) In [2(d)] the author proved: Let $w^{n}=D^{n}-d ; n \geqslant 2, D, d \in N ; D$ $\geqslant 2(n-1) d$. Then the J. P. A. of

$$
\begin{array}{r}
a^{(0)}=\left(a_{1}^{(0)}, \ldots, a_{n-1}^{(0)}\right), a_{s}^{(0)}=\sum_{i=0}^{s}\left(\begin{array}{c}
n-s-1+i \\
i
\end{array}\right) w^{s-i} D^{i} \\
(s=1, \ldots, n-1)
\end{array}
$$

is purely periodic and the length of the primitive period $m=n^{2}$ if $d \neq 1$ and $m=n$ if $d=1$.

(3) In [2(e)] the author proved: Let $w^{3}=D^{3}+3 D ; D \geqslant 2, D \in N$. Then the J. P. A. of $a^{(0)}=\left(w, w^{2}\right)$ becomes periodic; the lengths of the primitive preperiod and the primitive period are $l=m=4$.

(4) In [2(f)] the author proved: Let $w^{3}=D^{3}+6 D ; D=2 K, K=1,2$, ... Then the J. P. A. of $a^{(0)}=\left(w, w^{2}\right)$ is periodic; the length of the primitive preperiod is $l=4$, the length of the primitive period is $m=8$.

(5) In [3(a)] Hasse and the author proved: Let $P_{n}(x)=(x-D)\left(x-D_{1}\right)$ $\cdots\left(x-D_{n-1}\right)-d, n \geqslant 2, d \in N, D>D_{1}>\cdots>D_{n-1} ; D-D_{i} \geqslant$ $2 d(n-1)(i=1, \ldots, n-1) ; d|D, d| D_{i}(i=1, \ldots, n-1)$. Then $P_{n}(x)$ is irreducible over $Q$ and has $n$ real roots. Let $w, D<w<D+1$, be the largest of these $n$ roots. Let $P_{i i}=P_{i}=w-D_{i} \quad(i=1, \ldots, n-1) ; P_{i, k}=P_{i} P_{i+1} \cdots$ $P_{k}(1 \leqslant i<k \leqslant n-1)$. Let $a^{(0)}$ be a vector with the components

$$
a_{s}^{(0)}=d^{-1}(w,-D) P_{1} P_{2+s, n-1}(s=1, \ldots, n-3) ;
$$




$$
a_{n-2}^{(0)}=d^{-1}(w-D) P_{1}, \quad a_{n-1}^{(0)}=P_{1} .
$$

Then the J. P. A. of $a^{(0)}$ is purely periodic, and the length of its primitive period is $m=n(n-1)$ if $d \neq 1$, and $m=n-1$ if $d=1$.

Periodic J. P. A.'s are useful for approximating algebraic irrationals by rationals, as was shown by the author in $[2(\mathrm{~g})]$. Another important application is the calculation of units, as was mentioned in the. previous chapter. In [3(b)] Hasse and the author proved the

BAsic Theorem. Let the J. P. A. of $a^{(0)}=\left(a_{1}, \ldots, a_{n-1}\right)$ be periodic; let $l$ be the length of its primitive preperiod, $m$ the length of its primitive period; then

$$
e=\prod_{i=l}^{l+m-1} a_{n-1}^{(i)}
$$

is a unit in the field generated by the components of $a^{(0)}$ over $Q$.

By applying formula (1.1) to the periodic J. P. A. as outlined in (1) and (2) of this section the authors found that

$$
e_{k}=(w-D)^{-k}\left(w^{k}-D^{k}\right), \quad k \mid n, k>1,
$$

are units in the corresponding fields of degree $n$. The authors could not decide whether the $e_{k}$ belong to a system of independent units of the corresponding field.

By applying formula (1.1) to the periodic J. P. A. as outlined in (5) of this section, Hasse and the author found in [3(b)] that

$$
e_{k}=d^{-1}\left(w-D_{k}\right)^{n}, \quad k=1, \ldots, n-1,
$$

are units in the corresponding field and proved their independence.

In $[2(\mathrm{~h})]$ the author proved that, in the field $Q(w), w=D^{3}+k, D \in N$, $k \mid 3 D^{2}, e_{.}=k^{-1}(w-D)^{3}$ is a fundamental unit. He proved this independently, not using $(1,1)$. For $k=1$, the fundamental unit is $e_{1}=w-D$ with the exception $D=3$, where $e_{1}$ is the square of a fundamental unit. When $k=1$, and with the exception of $D=k=2$, where $e$ is the square of a fundamental unit, $e=k^{-1}(w-D)^{3}$ is always fundamental.

For $n=4,6$, Stender [9] proved that the units $e_{k}$ from (1.3) form a system of independent units.

The difficulty in calculating units from formula (1.1) stems, of course, from the necessity that the J.P. A. become periodic. But this happens very rarely, and apart from the few classes of infinitely many algebraic number fields, found by the author, no others are known. That the J.P. A. can be used for the calculation of units in infinitely many algebraic number fields, without knowing whether or not the corresponding J. P. A. becomes periodic, was proved by the author in [2(i)] 
where he stated:

THEOREM. Let $w$ be a real root of an nth degree polynomial and $Q(w)$ the algebraic number field generated by adjunction of $w$ to $Q$; let

$$
a^{(0)}=\left(a_{1}^{(0)}(w), a_{2}^{(0)}(w), \ldots, a_{n-1}^{(0)}(w)\right)
$$

be a vector whose components $a_{i}(w) \quad(i=1, \ldots, n-1)$ are algebraic integers in $Q(w)$. Let the components of the vectors $a^{(v)}(v=1,2, \ldots)$ in the J. P. A. of $a^{(0)}$ be rationalized, viz.

$$
\begin{aligned}
& a_{i}^{(v)}=\frac{C_{0, i}^{(v)}+C_{1, i}^{(v)} w+C_{2, i}^{(v)} w^{2}+\cdots+C_{n-1, i}^{(v)} w^{n-1}}{M_{v}} ; \\
& M_{v} \in N ; C_{j, i}^{(v)} \in \mathrm{Z}(j=0,1, \ldots, n-1 ; i=1, \ldots, n-1) .
\end{aligned}
$$

If, for a certain $v \geqslant 1, M_{v}=1$, then

$$
\begin{aligned}
e=\prod_{i=1}^{v} a_{n-1}^{(i)}= & A_{0}^{(v)}+a_{1}^{(v)} A_{0}^{(v+1)}+a_{2}^{(v)} A_{0}^{(v+2)} \\
& +\cdots+a_{n-1}^{(v)} A_{0}^{(v+n-1)},
\end{aligned}
$$

where the $A_{0}^{(v)}$ are obtained from the recursion formula

$$
\begin{aligned}
& A_{0}^{(0)}=1 ; A_{0}^{(1)}=A_{0}^{(2)}=\cdots=A_{0}^{(n-1)}=0 ; \\
& A_{0}^{(v+n)}=A_{0}^{(v)}+b_{1}^{(v)} A_{0}^{(v+1)}+\cdots+b_{n-1}^{(v)} A_{0}^{(v+n-1)} \\
& (v=0,1,2, \ldots)
\end{aligned}
$$

is a unit in $Q(w)$.

Of course, whether for every J. P. A. of $a^{(0)}=\left(a_{1}^{(0)}(w), \ldots, a_{n-1}^{(0)}(w)\right)$ there exists a $v$ such that $M_{v}=1$ could not be decided. But in [2(i)] he used formula (1.6) successfully in order to calculate a unit in $Q(w), w^{3}=m, m \in N$, $1<m<1000$, for almost all $m$. In [2(j)] the author has proved:

THEOREM. Let $m=a^{2}\left(a^{2} v^{3}+1\right)\left(a^{2} v^{3}+2\right), a, v \in N$; let $w^{3}=m$. Then in the J. P. A. of $a^{(0)}=\left(w, w^{2}\right)$, and with the notation (1.5), $M_{10}=1$, and $a$ unit is calculated from $e=A_{0}^{(10)}+a_{1}^{(10)} A_{0}^{(11)}+a_{2}^{(10)} A_{0}^{(12)}$, and its inverse has the form $e^{-1}=1-3 v\left(a^{2} v^{3}+1\right) w+3 v^{2} w^{2}$, which is a fundamental unit in $Q(w)$.

2. The J. P. A. of $a^{(0)}=\left(w, w^{2}\right), w^{3}=a^{6} T^{3}+3 T\left(a^{3} T+1\right)$. In this section we prove the first part of the main theorem of this paper as stated at the end of the introduction. We recall the hypotheses of this theorem: Let $m=$ $w^{3}=a^{6} T^{3}+3 T\left(a^{3} T+1\right) ; a, T \in N, Q(w)$ the real cubic algebraic number 
field generated by adjunction of $w$ to $Q$. Then the J. P. A. of $a^{(0)}=\left(w, w^{2}\right)$ is periodic: the length of the primitive preperiod is $l=4$, the length of the primitive period equals three.

PROOF. The J. P. A. of $a^{(0)}$ is the sequence $\left\langle a^{(v)}\right\rangle$, where the vectors $a^{(v)}$ $(v=1,2, \ldots)$ are obtained successively from $(0.2)$ by

$$
a^{(v+1)}=\left(\frac{a_{2}^{(v)}-b_{2}^{(v)}}{a_{1}^{(v)}-b_{1}^{(v)}}, \frac{1}{a_{1}^{(v)}-b_{1}^{(v)}}\right) \quad(v=0,1, \ldots),
$$

$$
b_{1}^{(v)}=\left[a_{1}^{(v)}\right] ; \quad b_{2}^{(v)}=\left[a_{2}^{(v)}\right] .
$$

In order to rationalize the denominator $a_{1}^{(v)}-b_{1}^{(v)}$ in $(2.1)$, the author used the formulas given in $[2(f)]$. Let

$$
\begin{aligned}
& a^{(v)}=\left(\frac{a_{v} w^{2}+b_{v} w+c_{v}}{M_{v}}, \frac{A_{v} w^{2}+B_{v} w+C_{v}}{M_{v}}\right) \\
& \quad(v=0,1, \ldots) ; m=w^{3} .
\end{aligned}
$$

Then the vector

$$
a^{(v+1)}=\left(\frac{a_{v+1} w^{2}+b_{v+1} w+c_{v+1}}{M_{v+1}}, \frac{A_{v+1} w^{2}+B_{v+1} w+C_{v+1}}{M_{v+1}}\right)
$$

is calculated by means of the recursive formulas

$$
\begin{gathered}
b_{v}^{2}-a_{v} c_{v}^{\prime}=M_{v} A_{v+1} ; \quad m a_{v}^{2}-b_{v} c_{v}^{\prime}=M_{v} B_{v+1} ; \\
c_{v}^{\prime 2}-m a_{v} b_{v}=M_{v} C_{v+1} ; \quad c_{v}^{\prime}=c_{v}-M_{v} b_{1}^{(v)} \\
m\left(a_{v} B_{v+1}+b_{v} A_{v+1}\right)+c_{v}^{\prime} C_{v+1}=M_{v} M_{v+1} ; \\
A_{v} C_{v+1}+B_{v} B_{v+1}+C_{v}^{\prime} A_{v+1}=M_{v} a_{v+1} ; \\
m A_{v} A_{v+1}+B_{v} C_{v+1}+C_{v}^{\prime} B_{v+1}=M_{v} b_{v+1} ; \\
m\left(A_{v} B_{v+1}+B_{v} A_{v+1}\right)+C_{v}^{\prime} C_{v+1}=M_{v} c_{v+1} ; \quad C_{v}^{\prime}=C_{v}-M_{v} b_{2}^{(v)}
\end{gathered}
$$

We shall now carry out the J. P. A. of $a^{(0)}=\left(w, w^{2}\right), w^{3}=m=a^{6} T^{3}+$ $3 T\left(a^{3} T+1\right)$, by means of formulas (2.3). We first need in order to find the vectors $b^{(v)}=\left(b_{1}^{(v)}, b_{2}^{(v)}\right)$ a good approximation for $w$ and $w^{2}$. We obtain from $w=a^{2} T\left[1+3 a^{-6} T^{-2}\left(a^{3} T+1\right)\right]^{1 / 3}$ the workable approximation

$$
\begin{aligned}
w & =a^{2} T+a^{-1}-\left(2 a^{3} T+1\right) a^{-10} T^{-3} \\
w^{2} & =a^{4} T^{2}+2 a T+a^{-2}-\left(2 a^{3} T+1\right) a^{-8} T^{-4} .
\end{aligned}
$$


We shall now write down the successive vectors $a^{(v)}$ and $b^{(v)} \quad(v=0,1, \ldots)$ without giving the calculations of every single operation-these would fill a whole book. The patient reader will enjoy verifying these results.

(2.5) $\quad a^{(0)}=\left(w, w^{2}\right)$.

(2.6) $\quad b^{(0)}=\left(a^{2} T, a^{4} T^{2}+2 a T\right)$.

$$
a^{(1)}=\left(\frac{-2 a T w^{2}+T\left(a^{3} T+3\right) w+a^{2} T^{2}\left(a^{3} T+3\right)}{3 T\left(a^{3} T+1\right)},\right.
$$

$$
\left.\frac{w^{2}+a^{2} T w+a^{4} T^{2}}{3 T\left(a^{3} T+1\right)}\right)
$$

(2.8) $\quad b^{(1)}=(0, a)$.

$$
a^{(2)}=\left(\frac{-a w^{2}+\left(2 a^{3} T+3\right) w-a^{2} T\left(a^{3} T+2\right)}{T\left(3 a^{6} T^{2}+10 a^{3} T+9\right)},\right.
$$

$$
\left.\frac{\left(a^{3} T+3\right) w^{2}+a^{2} T\left(a^{3} T+1\right) w+a T\left(a^{3} T+2\right)\left(a^{3} T+3\right)}{T\left(3 a^{6} T^{2}+10 a^{3} T+9\right)}\right):
$$

(2.10) $b^{(2)}=(0, a)$.

(2.11) $a^{(3)}=\left(w+a^{2} T, w^{2}+a^{2} T w+a T\left(a^{3} T+1\right)\right)$.

(2.12) $b^{(3)}=\left(2 a^{2} T, 3 a^{4} T^{2}+4 a T\right)$.

(2.13) $a^{(4)}=\left(\frac{-3 a T w^{2}+3 T w+3 a^{2} T^{2}\left(a^{3} T+2\right)}{3 T\left(a^{3} T+1\right)}, \frac{w^{2}+a^{2} T w+a^{4} T^{2}}{3 T\left(a^{3} T+1\right)}\right)$ :

(2.14) $b^{(4)}=(0, a)$.

(2.15) $a^{(5)}=\left(\frac{w-a^{2} T}{3 T\left(a^{3} T+1\right)}, \frac{w^{2}+a^{2} T w+a T\left(a^{3} T+3\right)}{3 T\left(a^{3} T+1\right)}\right)$.

(2.16) $b^{(5)}=(0, a)$.

(2.17) $a^{(6)}=\left(w+2 a^{2} T, w^{2}+a^{2} T w+a^{4} T^{2}\right)$.

(2.18) $b^{(6)}=\left(3 a^{2} T, 3 a^{4} T^{2}+3 a T\right)$.

(2.19) $a^{(7)}=a^{(4)}$.

By formula (2.19) the first part of our main theorem is completely proved. 
3. A fundamental unit in $Q(w)$. In this section we shall prove the second part of our main theorem, viz.: a fundamental unit in $Q(w), w^{3}=m=a^{6} T^{3}+$ $3 T\left(a^{3} T+1\right)$, is given by $e=1+a^{3} T-a w$. Since $M_{3}=1$ according to (2.11), we shall calculate a unit in $Q(w)$ by (1.6) which, in our cubic case, takes the form

$$
e_{1}=A_{0}^{(3)}+a_{1}^{(3)} A_{0}^{(4)}+a_{2}^{(3)} A_{0}^{(5)} \text {. }
$$

Since $b^{(1)}=b^{(2)}=(0, a)$, we calculate easily from $(1.7)$, for $n=3$,

$$
A_{0}^{(3)}=1 ; \quad A_{0}^{(4)}=a ; \quad A_{0}^{(5)}=a^{2} .
$$

Since, from (2.11), $a^{(3)}=\left(w+a^{2} T, w^{2}+a^{2} T w+a T\left(a^{3} T+1\right)\right)$, we obtain from (3.1)

$$
\begin{aligned}
& e_{1}=1+a\left(w+a^{2} T\right)+a^{2}\left(w^{2}+a^{2} T w+a T\left(a^{3} T+1\right)\right) \\
& e_{1}=\left(a^{3} T+1\right)^{2}+a\left(a^{3} T+1\right) w+a^{2} w^{2}
\end{aligned}
$$

From (3.3) we obtain

$$
\begin{aligned}
& e_{1}^{-1}= \frac{1}{\left(a^{3} T+1\right)^{2}+a\left(a^{3} T+1\right) w+a^{2} w^{2}} \\
&= \frac{a^{3} T+1-a w}{\left(a^{3} T+1\right)^{3}-a^{3} w^{3}}=a^{3} T+1-a w, \\
& e_{1}^{-1}=e=a^{3} T+1-a w .
\end{aligned}
$$

We shall also calculate a unit from formula (1.1) which in our case takes the form

$$
e_{2}=a_{2}^{(4)} a_{2}^{(5)} a_{2}^{(6)} \text {. }
$$

Substituting the values of $a_{2}^{(4)}, a_{2}^{(5)}, a_{2}^{(6)}$ from (2.13), (2.15), (2.17), we obtain, after some lengthy calculations,

$$
e_{2}=a^{2} w^{2}+a\left(a^{3} T+1\right) w+\left(a^{3} T+1\right)^{2}=e_{1},
$$

so that the formulas (1.1) and (1.6) lead to the same result. This does not always happen; as the author has shown in [2(i)], very often $e_{2}=e_{1}^{2}$.

We shall now show that $e=a^{3} T+1-a w$ is a fundamental unit in $Q(w)$, $N(e)=1$. Nagell [7] has proved that if a unit is of binary form as in (3.4), then it is either a fundamental unit or the square of a fundamental unit in the corresponding field. The reader will have no difficulties in verifying the inequalities

$$
0<e=a^{3} T+1-a w<1 .
$$

Presume $e$ is the square of a unit. Then, by Dirichlet (see e.g. [12]), 


$$
e^{3 / 2}=x+y w+z f^{-1} w^{2}, w^{3} \text { cubefree, } \quad w^{3}=f^{2} g,
$$

$$
w^{3} \neq \equiv \pm 1(\bmod 9), \quad f, g \in N ; x, y, z \in Z ;
$$

$$
\begin{aligned}
& e^{1 / 2}=1 / 3\left(x+y w+z f^{-1} w^{2}\right), w^{3} \text { cubefree, } w^{3}=f^{2} g, \\
& w^{3} \equiv \pm 1(\bmod 9), \quad f, g \in N, x, y, z \in \mathbf{Z} .
\end{aligned}
$$

In the first case of (3.8), $Q(w)$ is called a field of first kind, in the second casea field of second kind. The only interesting case is the second kind. The first kind need not be considered, since Nagell $[7$, p. 226] proved for this case that only for $m=20$ a square of a unit can be of the form $a+b w$. For fields of the second, we obtain from (3.8), putting $\rho=\exp 2 \pi i / 3$,

$$
\begin{aligned}
x+y w+z f^{-1} w^{2} & =3 e^{1 / 2}, \\
x+y w \rho+z f^{-1} w^{2} \rho^{2} & =3 e^{1 / 2}, \\
x+y w \rho^{2}+z f^{-1} w^{2} \rho & =3 e^{11 / 2} .
\end{aligned}
$$

From (3.9) we calculate $x, y, z$ :

$$
\begin{aligned}
& x=e^{1 / 2}+e^{11 / 2}+e^{\prime 1 / 2} \\
& y=w^{-1}\left(e^{1 / 2}+\rho^{2} e^{11 / 2}+\rho e^{\prime 1 / 2}\right), \\
& z=f w^{-2}\left(e^{1 / 2}+\rho e^{\prime 1 / 2}+\rho^{2} e^{\prime 11 / 2}\right) .
\end{aligned}
$$

Now $|e|<1$, $\left|e e^{\prime} e^{\prime \prime}\right|=1$, hence

$$
\left|e^{\prime}\right|=\left|e^{\prime \prime}\right|>1 \text {. }
$$

We further obtain $\left|e^{\prime}\right|=\left|a^{3} T+1-a w \rho\right| \leqslant a^{3} T+1+a w<a^{3} T+1+$ $a\left(a^{2} T+a^{-1}\right)$,

$$
\left|e^{\prime}\right|<2\left(1+a^{3} T\right)
$$

From (3.10), (3.11), (3.12) we now obtain

$$
|y|<w^{-1}\left(1+2\left(2\left(1+a^{3} T\right)^{1 / 2}\right)\right) \text {. }
$$

If $e$ were to be a square of a unit, we would obtain from the second equation of (3.8), $e=9^{-1}\left(x+y w+z f^{-1} w^{2}\right)^{2}$, hence, by comparison of coefficients of powers of $w$,

$$
\begin{aligned}
9\left(1+a^{3} T\right) & =x^{2}+2 f g y z \\
-9 a & =2 x y+g z^{2} \\
0 & =y^{2}+2 x z f^{-1} .
\end{aligned}
$$

We shall show that for fixed upper bounds for $a$ and $T, y=0$, so that from (3.14) also $z=0$, which is impossible. For $(a, T)=(1,1), m=7 \neq \pm 1(9)$. For $(a, T)=(1,2), m=26 \equiv-1(9)$. From $m=f^{2} g$ it follows in this case that 
$(f, g)=(1,26)$. From the first equation of (3.14) we obtain $27=x^{2}+52 y z$. The reader will easily verify from (3.14) (second and third equations) that $y z>0$, hence $27=x^{2}+52 y z$ is impossible. The reader should also note that for $m$ to be $\equiv \pm 1(9)$, neither $a$ nor $T$ can be a multiple of 3 . For $(a, T)=(2,1), m=$ $91,(f, g)=(1,91)$, and from $(3.14), 81=x^{2}+182 y z$, which is also impossible for $y z>0$. The next smallest value is assumed by $m$ for $(a, T)=(1,5), m=$ $215 \equiv-1(9)$. We shall therefore presume in our further investigations that $m \geqslant$ 215. We now obtain from (3.13): $|y|<w^{-1}+\left(2\left(2\left(1+a^{3} T\right) w^{-2}\right)^{1 / 2}\right)$. From (2.4) $w^{2}>a^{4} T^{2}+2 a T+1$, hence

$$
|y|<w^{-1}+2\left(2 a^{-1} T^{-1}\right)^{1 / 2} .
$$

Since $w^{3}=m \geqslant 215, w \geqslant 215^{1 / 3}$, and in view of having substantially increased the second summand on the right side of $|y|$, we can accept the proper approximation $w \geqslant 216^{1 / 3}=6$, hence from (3.15) $|y|<6^{-1}+2\left(2 a^{-1} T^{-1}\right)^{1 / 2}$. We are looking for those values of $(a, T)$, for which $\left(2 a^{-1} T^{-1}\right)^{1 / 2}<5 / 12$. This is equivalent to

$$
a T>288 / 25 \geqslant 11
$$

Having excluded the cases $(a, T)=(1,1),(1,2),(2,1)$, and taking into account that $a$ and $T$ are not multiples of 3 and that $m$ is congruent $\pm 1(9)$, this leaves us with the following cases:

$$
\begin{array}{lll}
a=1, & T=5, & m=215 \quad-1(9), \\
a=1, & T=11, & m=1727 \quad-1(9), \\
a=2, & T=4, & m=4492 \quad 1(9), \\
a=4, & T=2, & m=33542 \quad-1(9), \\
a=5, & T=1, & m=16003 \quad 1(9), \\
a=8, & T=1, & m=263683 \quad 1(9), \\
a=11, T=1, & m=1775557 \quad 1(9) .
\end{array}
$$

We have the factorization

$$
\begin{aligned}
215 & =5.43, \\
1727 & =11.157, \\
4492 & =2^{2} .1123, \\
33542 & =2.31 .541, \\
16003 & =13.1231, \\
263683 & =7.139 .271, \\
1775557 & =7.253651 .
\end{aligned}
$$

In all these cases, with the exception of 4492 , we obtain $f=1, g=m$. From the first equation of (3.14) we therefore obtain

$$
9\left(1+a^{3} T\right)=x^{2}+2\left(a^{6} T^{3}+3 T\left(1+a^{3} T\right)\right) y z
$$


which is impossible for the values of $(a, T)$ in (3.17) and with $y z>0$. For $m=$ 4492, $f=2, g=1123$, and we obtain from (3.14) $9\left(1+a^{3} T\right)=x^{2}+m y z=$ $x^{2}+4492 y z$, or $9(1+8.4)=x^{2}+4492 y z$, which is also impossible. Thus the proof that $e$ is a fundamental unit is completed.

Concluding, we shall still show that there exist only a finite number of values for $a$ and $T$, hence of $m$, for which $m$ is not cubefree. We have, presuming the opposite, $a^{6} T^{3}+3 a^{3} T^{2}+3 T=t u^{3}$, or, multiplying both sides by $a^{3}, a^{9} T^{3}+$ $3 a^{6} T^{2}+3 a^{3} T+1-1=t a^{3} u^{3},\left(a^{3} T+1\right)^{3}-t z^{3}=1, v^{3}-t z^{3}=1 ; a^{3} T+$ $1=v, a u=z$. Now, by the Nagell's theorem [7], which is a special case of the Thue-Siegel theorem, $v^{3}-t z^{3}=1$ has at most one solution for a fixed $t$. This proves our claim. In the same way it is easily verified that $m$ cannot be a perfect cube. In this case $t=1, v^{3}-z^{3}=1$ which has only the trivial solutions $(v, z)=$ $(1,0)$ or $(0,-1)$.

\section{BIBLIOGRAPHY}

1a. G. Bergmann, Untersuchungen zur Einheitsgruppe in den totalkomplexen algebraischen Zahlkörpern sechsten Grades (Über P) im Rahmen der 'Theorie der Netze", Math. Ann. 161 (1965), 349-364. MR 34 \#1299,

1b. - Zur numerischen Bestimmung einer Einheitenbasis, Math. Ann. 166 (1966), 103-105. MR 34 \#1300.

143-168. MR 35 \#158.

1c. - Beispiele numerischer Einheitenbestimmung, Math. Ann. 167 (1966),

2a. L. Bernstein, The Jacobi-Perron algorithm-Its theory and application, Lecture Notes in Math., vol. 207, Springer-Verlag, Berlin and New York, 1971 . MR 44 \#2696.

2b. - The modified algorithm of Jacobi-Perron, Mem. Amer. Math. Soc. No. 67 (1966). MR 34 \#5274.

2c. - Periodical continued fractions for irrationals of degree $n$ by Jacobi's algorithm, J. Reine Angew. Math. 213 (1963/64), 31-38. MR 27 \#5727; 30, p. 1204.

2d. - New infinite classes of periodic Jacobi-Perron algorithms, Pacific J. Math. 16 (1966), 439-469. MR 32 \#7505.

2e. - Periodicity of Jacobi's algorithm for a special type of cubic irrationals, J. Reine Angew. Math. 213 (1963/64), 137-146. MR 29 \#3434.

2f. - A 3-dimensional periodic Jacobi-Perron algorithm of period length $8, \mathrm{~J}$. Number Theory 4 (1972), 48-69. MR 45 \#3328.

2g. - Rational approximations of algebraic irrationals by means of a modified Jacobi-Perron algorithm, Duke Math. J. 32 (1965), 161-176; correction, ibid. 32 (1965), 765. MR 31 \#123; 32 \#92.

2h. - On units and fundamental units, J. Reine Angew. Math. 257 (1972), 129-145. MR 48 \#2111.

2i. - Einheitenberechnung in Kubischen Körpern mittels des Jacobi-Perronschen Algorithmus aus der Rechenanlage, J. Reine Angew. Math. 244 (1970), 201-220. MR 42 \#5947.

2j. - Fundamental units from the preperiod of a generalized Jacobi-Perron algorithm, J. Reine Angew. Math. (in print).

3a. L. Bernstein and H. Hasse, An explicit formula for the units of an algebraic number field of degree $n>2$, Pacific J. Math. 30 (1969), 293-365. MR 40 \#120.

3b. - Einheitenberechnung mittels des Jacobi-Perronschen Algorithmus, J. Reine Angew. Math. 218 (1965), 51-69. MR 31 \#4765.

4. K. K. Billevič, On units of algebraic fields of third and fourth degree, Mat. Sb. 40 (82) (1956), 123-136. (Russian) MR 19, 533. 
5. C. G. J. Jacobi, Allgemeine Theorie der kettenbruchaehnlichen Algorithmen, in welchen jede Zahl aus drei vorhergehenden gebildet wird, J. Reine Angew. Math. 69 (1869), 29-64.

6. H. London and R. Finkelstein, On Mordell's equation $Y^{2}-k=x^{3}$, Bowling Green State Univ. Press, Bowling Green, Ohio, 1973.

7. T. Nagell, Solution complète de quelque équation cubique à deux indéterminées, J. Math. Pures Appl. (9) 4 (1925), 209-270.

8. O. Perron, Grundlagen für eine Theorie des Jacobischen Kettenbruchalgorithmus, Math. Ann. 64 (1907), 1-76.

9a. H.-J. Stender, Grundeinheiten für einige unendliche Klassen reiner biquadratischer Zahlkörper mit einer Anwendung auf die diophantische Gleichung $x^{4}-a y^{4}= \pm c(c=1,2$, 4, oder 8), J. Reine Angew. Math. 264 (1973), 207-220. MR 48 \#1 1040.

9b. - Über die Einheitengruppe der reinen algebraischen Zahlkörper sechsten Grades, J. Reine Angew. Math. 269 (1974), 78-93.

10. G. F. Voronor, On a generalization of the algorithm of continued fractions, Doctoral Dissertation, Warsaw, 1896. (Russian)

11. H. Zassenhaus, On the units of orders, J. Algebra 20 (1972), 368-395. MR 44 \#6659.

12. R. Dedekind, Über die Anzahl der Idealklassen in reinen kubischen Zahlkörpern, J. Reine Angew. Math. 121 (1900), 40-123.

DEPARTMENT OF MATHEMATICS, ILLINOIS INSTITUTE OF TECHNOLOGY, CHICAGO, ILLINOIS 60616 